\title{
The potential of Cosmos sulphureus flower extract as a bioherbicide for Cyperus rotundus
}

\author{
DYAH WENY RESPATIE ${ }^{1, \boldsymbol{v}}$, PRAPTO YUDONO ${ }^{1}$, AZIZ PURWANTORO ${ }^{1}$, Y. ANDI TRISYONO ${ }^{2}$ \\ ${ }^{1}$ Department of Agronomy, Faculty of Agriculture, Universitas Gadjah Mada. Jl. Flora No. 1, Bulaksumur, Sleman 55281, Yogyakarta, Indonesia. \\ Tel./fax.: +62-274-563062, `email: wenyrespatie@ugm.ac.id \\ ${ }^{2}$ Department of Crop Protection, Faculty of Agriculture, Universitas Gadjah Mada. Jl. Flora No. 1, Bulaksumur, Sleman 55281, Yogyakarta, Indonesia
}

Manuscript received: 3 October 2019. Revision accepted: 16 November 2019.

\begin{abstract}
Respatie. D. W, Yudono P, Purwantoro A, Trisyono Y.A. 2019. The potential of Cosmos sulphureus flower extract as a bioherbicide for Cyperus rotundus. Biodiversitas 20: 3568-3574. Cosmos (Cosmos sulphureus Cav.) flower is recognized as natural source of bioherbicide compounds for several weeds. The purple nutsedge (Cyperus rotundus L.) is an important weed, and this research was aimed to determine the effects of cosmos flower extract on this weed. Dried cosmos flowers were threshed and extracted using maceration method with $70 \%$ alcohol. The experiment was conducted in the greenhouse using a completely randomized design (CRD) with three replications. Purple nutsedge tubers were planted in polybags and treated with cosmos flower extract applicated once to three times with $300 \mathrm{~mL}^{\text {polybag }}{ }^{-1}$ at the concentration of $40 \%$. Different levels of inhibitions on purple nutsedge growth were observed at 30 and 60 days after sowing (DAS) due to the presence of gallic acid in the extract. Growth parameters for purple nutsedge were analyzed using Analysis of Variance (ANOVA) and followed by Least Significant Differences (LSD) at $\alpha=0.05$. Significant reductions in the number of mother shoots, daughter shoots, and roots, length of mother leaves, length of rhizomes, root, and total leaf area of the purple nutsedge treated with the cosmos flower extract at 30 DAS compared to those in the control. A significant reduction was also observed in foliage dry weight, underground organs dry weight, and total dry weight of purple nutsedge. The three application times yielded maximum inhibition. In addition, these treatments reduced $\mathrm{N}, \mathrm{P}$, and $\mathrm{K}$ content, chlorophyll content, and photosynthesis rates at 30 DAS. These results suggest that cosmos flower extract has the potential for controlling purple nutsedge.
\end{abstract}

Keywords: Allelopathy, bioherbicides, Cosmos sulphureus, Cyperus rotundus, gallic acid

Abbreviations: DAS: days after showing, BAW: butanol, acetic acid, water, CRD: completely randomized design, TLC: Thin Layer Chromatography, DW: dry weight

\section{INTRODUCTION}

Purple nutsedge (Cyperus rotundus L.) is a perennial weed with detrimental impact on agriculture and is widely spread throughout the world (Holm et al. 1991; Horowitz 1992; Webster and Grey, 2014). Purple nutsedge reproduces by vegetative propagation using basal bulbs and tubers to proliferate into many rhizomes, resulting in populations to grow rapidly and causing great loss in agricultural yields (Nishimoto 2001; Hussain et al. 2017). Studies have reported yield reduction up to $80 \%$ in soybean (Moenandir 1993; Hendrival et al. 2014; Yadav et al. 2017) and $97 \%$ in rice (Arunbabu and Jena 2018). Thus, proper management of this weed is essential which has heavily relied on manual, mechanical weeding, and synthetic herbicides, such as glyphosate, halosulfuron and imizaqueen (Ferell et al. 2004; Durigan et al. 2006; ElRokiek et al. 2006; El-Rokiek et al. 2007; El-Rokiek et al. 2009; Hussain 2011; Webster and Grey 2014). The continuous use of chemical herbicides can cause detrimental effects on environmental and human health. Therefore, there is merit in exploring other control options that are more environmentally friendly and effective, such as using allelopathic compounds as bioherbicides.
Allelopathy refers to the harmful effects of secondary metabolites produced by plants on the growth and development of other organisms (Hussain et al. 2017). A vast number of researches have reported Asteraceae plants to be a source of allelochemical compounds, such as phenolic acids and terpenes (Batish et al. 2007; Khan and Khan 2010; Shafique et al. 2011; Arora et al. 2015; Wichittrakarn 2015). The allelochemical phenol inhibits plant growth by altering cell membrane permeability. This process inhibits nutrient absorption and eventually affects synthesis of endogenous plant hormones, various functions and activities of enzymes, photosynthesis, protein synthesis, plant cell division and elongation (Abenavoli et al. 2003; Li et al. 2010). In addition, phenolics are able to reduce chlorophyll levels by inhibiting chlorophyll synthesis or inducing chlorophyll degradation (Yang et al. 2004; Shankar et al. 2014). Due to these mechanisms, allelochemicals are widely used as bioherbicide in agricultural fields (Bogatek et al. 2006; Batish et al. 2007; Albuquerque et al. 2011; Cheng and Cheng 2015; Sangeetha and Baskar 2015).

Asteraceae are potential sources of natural herbicides due to their content of noticeable phenolic allelochemicals, such as gallic acid, ferulic acid, p-coumarin and flavonoids 
(Shafique et al. 2011). Cosmos (Cosmos sulphureus), an Asteraceae species, is a common ornamental plant worldwide and well known to contains phenolic acids, flavonoids, and anthocyanin; In addition, cosmos is also used as refuge plants in agricultural system which provide breeding spots and additional nutrition to beneficial organisms, such as natural enemies against insect pests (Kaur et al. 2006; Zeng et al. 2008; Kaisoon et al. 2011; Sugiharti et al. 2018). In rural areas where mechanical and chemical control options might be expensive or, manual weeding may a laborious option, utilizing available resources is essential. Therefore, there is a potential to use cosmos as a source for bioherbicide and merit to observe its bioherbicidal properties on the purple nutsedge. The specific objectives of this study were to determine chemical content of cosmos flower extracts and its effects on several purple nutsedge growth and physiological parameters.

\section{MATERIALS AND METHODS}

\section{Origin of the cosmos and purple nutsedge}

Several three-month-old cosmos were collected from a field at Banguntapan Village, Bantul District, Special Region of Yogyakarta Province, Indonesia (07 48' 17" S and $110^{\circ} 24^{\prime} 45^{\prime \prime}$ E) in December 2017. Purple nutsedge used as a source of tubers were obtained from Pesona Pengklik Pantai Samas, Srigading Village, Kulon Progo District, Special Region of Yogyakarta Province, Indonesia $\left(08^{\circ} 00^{\prime} 15,7^{\prime \prime} \mathrm{S}\right.$ and $\left.110^{\circ} 16^{\prime} 12,9^{\prime \prime} \mathrm{E}\right)$ in February 2018. The references compound for qualitative assessment were vanillic acid, caffeic acid, dihydroxybenzoic acid, coumaric acid, gallic acid, and syringic acid. These compounds were purchased from Sigma-Aldrich Chemicals (St. Louis, MO, USA).

\section{Determination of gallic acid \\ Preparation of solution}

Cosmos flowers were dried to constant weight in an electric oven at $40^{\circ} \mathrm{C}$ for 48 hours. Dried flowers were collected then ground, threshed to powder, and then stored in polythene bags. Dried flower powder $(2.5 \mathrm{~g})$ was extracted using the maceration method with $70 \%$ ethanol $(50 \mathrm{ml})$. After 24 hours, it was filtered using muslin cloth $(<0.1 \mathrm{~mm}$ diameter) followed by Whatman filter paper No. 1. The filtrate was then evaporated with nitrogen gas to form a thick extract and stored in the refrigerator.

\section{Qualitative assessment}

Thin Layer Chromatography (TLC) was used to determine the content of cosmos flower extracts compared with six reference compounds (vanillic acid, caffeic acid, dihydrobenzoic, coumaric acid, gallic acid, and syringic acid) widely known as allelochemicals. Twenty $\mu$ l of cosmos flower extracts and the reference compounds were spotted on the origin of the $\mathrm{F}_{254}$ silica TLC gel (still phase) and the plate was placed in the Camag chamber saturated with a 9:3:1 butanol, acetate acid, and water solvent (BAW). After 1 hour, the plate was removed, air dried, and observed under ultraviolet light at the absorbance level of
$254 \mathrm{~nm}$. Stains found in the plate indicated that the extract might contain phenolic acid. The plate was sprayed with $\mathrm{FeCl}_{3}$ and dried in an oven at $105^{\circ} \mathrm{C}$ for 3 minutes. If the stains turned blue, it was confirmed that the flower extract contained phenolic acids. The Rf values were calculated to identify the content of extract by comparing it with the reference of the compound.

\section{Quantitative assessment}

Dried flower powder $(2.5 \mathrm{~g})$ was extracted with $50 \mathrm{ml}$ ethanol. The extract was filtered, and concentrated. Ethanol was then added to $500 \mu \mathrm{l}$ volumetric flasks. Twenty $\mu \mathrm{l}$ solution was subjected to TLC for simultaneous gallic acid estimation. Ascending development, migration distance (80 $\mathrm{mm}$ ) was performed at $25^{\circ} \mathrm{C}$ with toluene: methanol-formic acid (95:5) as a mobile phase for gallic acid in a Camag chamber previously saturated for 30 minutes. Plate was then dried at $50^{\circ} \mathrm{C}$ in an oven for 5 minutes. Densitometric scanning was performed with the absorbance value at 280 $\mathrm{nm}$ to determine the content of gallic acid.

\section{Effect of cosmos flower extracts on the growth and development of purple nutsedge \\ Extract preparations}

Cosmos flowers were dried to constant weight in an electric oven at $40^{\circ} \mathrm{C}$ for 48 hours. Dried flowers were collected then ground, threshed to powder, and then stored in polythene bags. Forty grams of powder were passed through a $1.5 \mathrm{~mm}$ mesh and transferred to a labeled bottle. One hundred $\mathrm{ml}$ of sterile and deionized distilled water was added to the bottle and left at room temperature $( \pm 25$ $26^{\circ} \mathrm{C}$ ). After 24 hours, the material was filtered to obtain the extract of $40 \%$. Solutions were then extracted again through the muslin cloth $(<0.1 \mathrm{~mm}$ diameter) followed by Whatman filter paper No. 1.

\section{Bioassays}

Experiment was conducted in the greenhouse using a completely randomized design (CRD) with three replications. Four purple nutsedge dormant tubers with uniform weight $( \pm 5 \mathrm{~g})$ were planted in polybags with a diameter of $30 \mathrm{~cm}$ filled with $3 \mathrm{~kg}$ of sterile soil. The treatments were three different regimes of the cosmos flower extract application i.e. once (at the planting time), 2 times (at the planting time and 1-week after planting) and 3 times (at the planting time, 1-week and 2-week after planting). The control was treated with distilled water 3 times (at the planting time, 1-week and 2-week after planting). Each application used $300 \mathrm{~mL}$ of the extract or the distilled water for each experimental unit. There were 12 experimental units for each treatment. Two plants were collected from each experimental unit of treatments at 30 and 60 days after sowing (DAS) for observations.

\section{Observations \\ Growth parameters}

Growth parameters measured in this experiment were mother shoots or tuber numbers, leaves numbers of mother shoots/tuber, length of mother leaves $(\mathrm{cm})$, daughter shoots/tuber numbers, leaves numbers of daughter 
shoots/tuber, rhizomes/tuber numbers, rhizomes/tuber length, the number of propagative organs/tuber (basal bulb and tubers)/plant, foliage dry weight (g/plant), underground organs dry weight (g/plant), and total dry weight (DW) (g/plant).

\section{Nutrient content of purple nutsedge}

Nitrogen contents were determined using Kjeldahl methods. Phosphor contents were determined using Spektrofotometer Spectronic 21D and potassium contents were determined using a flame photometer AAS Shimadzu 6200 .

\section{Chlorophyll content of purple nutsedge}

Chlorophyll was extracted using $80 \%$ acetone and quantified following Kambe et al. (2015). Optical density of the supernatant was measured using a spectrophotometer (Guha et al. 2009) at 663 and $645 \mathrm{~nm}$. Chlorophyll a, b and total chlorophyll were calculated using the following formulas:

$$
\begin{aligned}
& \text { Chlorophyll a }\left(\mathrm{mg} \mathrm{g}^{-1}\right)=\frac{[12.7(D 663)-2.69(D 645)] \times V}{1000 \times W} \\
& \text { Chlorophyll } b\left(\mathrm{mg} \mathrm{g}^{-1}\right)=\frac{[22.9(\mathrm{D} 663)-4.68(\mathrm{D} 645)] \times \mathrm{V}}{1000 \times \mathrm{W}} \\
& \text { Total Chlorophyll }\left(\mathrm{mg} \mathrm{g}^{-1}\right)=\frac{[8.02(\mathrm{D} 663)-20.2(\mathrm{D} 645)] \times V}{1000 \times \mathrm{W}}
\end{aligned}
$$

Where:

D: Optical density at respective $\mathrm{nm}$;

V: Final volume of chlorophyll extract in $80 \%$ acetone;

$\mathrm{W}$ : Fresh weight of the tissue extracted.

\section{Photosynthetic rate of purple nutsedge}

Photosynthetic rates were determined using a photosynthetic analyzer Licor- 6400.

\section{Statistical analysis}

Data were checked for normality and homogeneity using Shapiro-Wilk and Bartlett's test, respectively. Growth parameters for purple nutsedge were analyzed using Analysis of Variance (ANOVA) and followed by Least Significant Differences (LSD) at the 0.05 probability level. Statistical analyses used $R$ statistical software v.3.2.2 using the agricolae package.

\section{RESULTS AND DISCUSSION}

\section{Determination of gallic acid}

Analysis using TLC demonstrated that the cosmos flower extracts contained a phenolic compound similar to gallic acid based on its Rf values of 0.86 (Figure 1). Follow up analysis revealed that the content of gallic acid in the

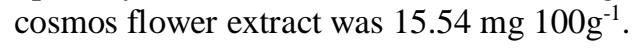

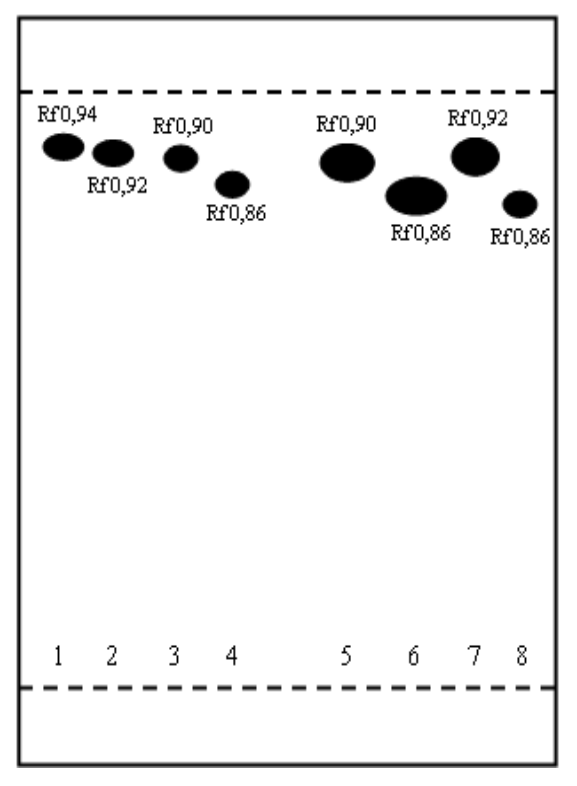

Figure 1. Rf values of cosmos flower extract (4 and 8) and six reference compounds on F254 TLC plate; (i) vanillic acid; (ii) caffeic acid; (iii) dihydrobenzoic; (vi) coumaric acid; (v) gallic acid; and (vi) syringic acid. The figure was drawn based on the result from the TLC plate.

\section{Effect of cosmos flower extracts on the growth and development of purple nutsedge \\ Growth parameters}

Purple nutsedge underground organs responded differently to the application frequencies of cosmos flower extract. Purple nutsedge rhizomes/tuber length, root length and root numbers of the purple nutsedge treated with the cosmos flower extract were significantly lower compared to those in the control and decreased correspondingly with the increase of application frequencies at 30 DAS (Table 1). All purple nutsedge growth parameters of underground organs were not significantly different at 60 DAS except for the root numbers (Figure 2). Root numbers were only significantly different compared to those in the control at 3 application times.

Mother shoots/tuber numbers, mother leaves length, and total leaf area of the purple nutsedge treated with the cosmos flower extract was significantly lower compared with the control and the impacts became more prominent as the application frequency increased at 30 DAS (Table 2). All foliage growth parameters were not significantly different compared with the control at 60 DAS (Figure 2). The extract induced significant reductions in growth of mother shoots/tuber and daughter shoots.

\section{Nutrient content}

$\mathrm{N}$, P, and $\mathrm{K}$ levels were significantly reduced in the purple nutsedge treated with the cosmos flower extract compared with the control at 30 DAS (Table 3). The level of reduction increased correspondingly as the application frequencies increased with the maximum reduction at 3 application times. $\mathrm{N}$ content was the only nutrient to be significantly lower than in the content at 60 DAS after application. 
Table 1. Growth of purple nutsedge underground organs treated with different application times of cosmos flower extract.

\begin{tabular}{|c|c|c|c|c|c|c|}
\hline \multirow{2}{*}{$\begin{array}{l}\text { Growth } \\
\text { parameters }\end{array}$} & \multirow[b]{2}{*}{ DAS } & \multicolumn{4}{|c|}{ Application times } & \multirow[b]{2}{*}{ F-value; $\mathbf{d f}_{\text {denum }} ; P$} \\
\hline & & Control & 1 & 2 & 3 & \\
\hline Rhizomes/tuber & 30 & $1.33 \pm 0.17 \mathrm{a}$ & $1.50 \pm 0.50 \mathrm{a}$ & $0.83 \pm 0.17 \mathrm{a}$ & $1.83 \pm 0.60 \mathrm{a}$ & F-value $=1.042 ; \mathrm{df}_{\text {denum }}=8 ; \mathrm{P}=0.425$ \\
\hline number & 60 & $5.17 \pm 0.17 \mathrm{a}$ & $4.17 \pm 0.44 \mathrm{a}$ & $3.83 \pm 0.44 \mathrm{a}$ & $3.33 \pm 1.36 \mathrm{a}$ & F-value $=1.053 ; \mathrm{df}_{\text {denum }}=8 ; \mathrm{P}=0.421$ \\
\hline Rhizomes/tuber & 30 & $6.68 \pm 0.91 \mathrm{a}$ & $6.08 \pm 0.75 \mathrm{ab}$ & $6.62 \pm 0.89 \mathrm{ab}$ & $4.53 \pm 0.19 b$ & F-value $=2.294 ; \mathrm{df}_{\text {denum }}=8 ; \mathrm{P}=0.0155$ \\
\hline length $(\mathrm{cm})$ & 60 & $6.72 \pm 0.58 \mathrm{a}$ & $7.39 \pm 0.83 a$ & $7.07 \pm 0.29 \mathrm{a}$ & $5.07 \pm 0.95 \mathrm{a}$ & $\mathrm{F}$-value $=2.103 ; \mathrm{df}_{\text {denum }}=8 ; \mathrm{P}=0.178$ \\
\hline Propagative & 30 & $0.33 \pm 0.33 \mathrm{a}$ & $0.00 \pm 0.00 \mathrm{a}$ & $0.00 \pm 0.00 \mathrm{a}$ & $0.00 \pm 0.00 \mathrm{a}$ & F-value $=1,000 ; \mathrm{df}_{\text {denum }}=8 ; \mathrm{P}=0.441$ \\
\hline organs/tuber & 60 & $3.17 \pm 0.44 \mathrm{a}$ & $2.83 \pm 0.44 a$ & $2.50 \pm 0.29 \mathrm{a}$ & $2.33 \pm 0.83 \mathrm{a}$ & F-value $=0.468 ; \mathrm{df}_{\text {denum }}=8 ; \mathrm{P}=0.713$ \\
\hline
\end{tabular}

(basal bulb and

tubers)/plant

number

$\begin{array}{lcccccc}\text { Root length } & 30 & 31.52 \pm 5.76 \mathrm{a} & 34.97 \pm 1.52 \mathrm{ab} & 28.57 \pm 4.47 \mathrm{ab} & 17.72 \pm 3.23 \mathrm{~b} & \text { F-value }=2.941 ; \mathrm{df}_{\text {denum }}=8 ; \mathrm{P}=0.0489 \\ (\mathrm{~cm}) & 60 & 32.13 \pm 2.23 \mathrm{a} & 36.82 \pm 4.97 \mathrm{a} & 24.75 \pm 5.27 \mathrm{a} & 26.52 \pm 5.36 \mathrm{a} & \text { F-value }=1.428 ; \mathrm{df}_{\text {denum }}=8 ; \mathrm{P}=0.305 \\ \text { Root number } & 30 & 22.17 \pm 3.68 \mathrm{a} & 17.67 \pm 0.88 \mathrm{ab} & 11.67 \pm 1.17 \mathrm{bc} & 10.00 \pm 1.44 \mathrm{c} & \text { F-value }=7.063 ; \mathrm{df}_{\text {denum }}=8 ; \mathrm{P}=0.0123\end{array}$

$\begin{array}{lllllll}\text { Root number } & 30 & 22.17 \pm 3.68 \mathrm{a} & 17.67 \pm 0.88 \mathrm{ab} & 11.67 \pm 1.17 \mathrm{bc} & 10.00 \pm 1.44 \mathrm{c} & \text { F-value }=7.063 ; \mathrm{df}_{\mathrm{denum}}=8 ; \mathrm{P}=0.0123 \\ & 60 & 30.83 \pm 5.73 \mathrm{a} & 23.67 \pm 2.19 \mathrm{ab} & 20.00 \pm 1.04 \mathrm{ab} & 17.50 \pm 5.03 \mathrm{~b} & \text { F-value }=2.103 ; \mathrm{df}_{\mathrm{denum}}=8 ; \mathrm{P}=0.0178\end{array}$

Note: means \pm SE followed by the same letter in the same rows were not significantly different according to LSD $\alpha=0.05$. $1=$ at the planting time; $2=$ at the planting time and 1 -week after planting; $3=$ at the planting time, 1 -week and 2 -week after planting. The concentration of extract was $40 \%$.

Table 2. Growth of purple nutsedge foliage treated with different application times of cosmos flower extract

\begin{tabular}{|c|c|c|c|c|c|c|}
\hline \multirow{2}{*}{$\begin{array}{l}\text { Growth } \\
\text { parameters }\end{array}$} & \multirow{2}{*}{ DAS } & \multicolumn{4}{|c|}{ Application times } & \multirow{2}{*}{ F-value; $\mathbf{d f}_{\text {denum }} ; P$} \\
\hline & & Control & 1 & 2 & 3 & \\
\hline Number of & 30 & $3.50 \pm 0.50 \mathrm{a}$ & $2.50 \pm 0.29 \mathrm{a}$ & $2.00 \pm 0.29 b$ & $1.67 \pm 0.17 \mathrm{~b}$ & F-value $=5.750 ; \mathrm{df}_{\text {denum }}=8 ; \mathrm{P}=0.0214$ \\
\hline $\begin{array}{l}\text { mother shoots / } \\
\text { tuber }\end{array}$ & 60 & $3.67 \pm 0.83 \mathrm{a}$ & $2.83 \pm 0.17 \mathrm{a}$ & $3.83 \pm 0.60 \mathrm{a}$ & $2.50 \pm 0.50 \mathrm{a}$ & F-value $=1.243 ; \mathrm{df}_{\text {denum }}=8 ; \mathrm{P}=0.357$ \\
\hline Number of leaves & 30 & $24.83 \pm 1.69 \mathrm{a}$ & $18.17 \pm 2.24 b$ & $13.67 \pm 1.74 b c$ & $11.00 \pm 1.04 \mathrm{c}$ & F-value $=12.20 ; \mathrm{df}_{\text {denum }}=8 ; \mathrm{P}=0.00236$ \\
\hline $\begin{array}{l}\text { of mother shoots/ } \\
\text { tuber }\end{array}$ & 60 & $37.17 \pm 6.09 \mathrm{a}$ & $27.33 \pm 1.20 \mathrm{a}$ & $35.00 \pm 3.00 \mathrm{a}$ & $25.83 \pm 4.92 \mathrm{a}$ & F-value $=1.744 ; \mathrm{df}_{\mathrm{der}}$ \\
\hline Length of mother & 30 & $48.42 \pm 0.48 \mathrm{a}$ & $44.78 \pm 2.45 \mathrm{a}$ & $41.80 \pm 0.94 \mathrm{a}$ & $35.10 \pm 3.11 b$ & F-value $=7.578 ; \mathrm{df}_{\text {denum }}=8 ; \mathrm{P}=0.0101$ \\
\hline leaves $(\mathrm{cm})$ & 60 & $64.20 \pm 3.67 \mathrm{a}$ & $61.23 \pm 2.47 \mathrm{a}$ & $66.92 \pm 2.64 \mathrm{a}$ & $61.07 \pm 1.03 \mathrm{a}$ & F-value $=1.117 ; \mathrm{df}_{\text {denum }}=8 ; \mathrm{P}=0.398$ \\
\hline Number of & 30 & $1.33 \pm 0.44 \mathrm{a}$ & $0.67 \pm 0.33 \mathrm{ab}$ & $0.50 \pm 0.00 \mathrm{ab}$ & $0.33 \pm 0.17 b$ & F-value $=2.306 ; \mathrm{df}_{\text {denum }}=8 ; \mathrm{P}=0.050$ \\
\hline $\begin{array}{l}\text { daughter shoots / } \\
\text { tuber }\end{array}$ & 60 & $4.33 \pm 0.73 \mathrm{a}$ & $3.17 \pm 0.73 \mathrm{a}$ & $2.67 \pm 0.44 \mathrm{a}$ & $2.67 \pm 1.17 \mathrm{a}$ & F-value $=0.947 ; \mathrm{df}_{\text {denum }}=8 ; \mathrm{P}=0.463$ \\
\hline $\begin{array}{l}\text { Number of leaves } \\
\text { of daughter } \\
\text { shoots / tuber }\end{array}$ & $\begin{array}{l}30 \\
60\end{array}$ & $\begin{array}{c}3.33 \pm 1.77 \mathrm{a} \\
20.17 \pm 6.19 \mathrm{a}\end{array}$ & $\begin{array}{c}2.50 \pm 0.87 \mathrm{a} \\
12.17 \pm 2.62 \mathrm{a}\end{array}$ & $\begin{array}{l}3.33 \pm 1.77 \mathrm{a} \\
11.83 \pm 1.45 \mathrm{a}\end{array}$ & $\begin{array}{c}1.67 \pm 0.44 \mathrm{a} \\
12.17 \pm 4.66 \mathrm{a}\end{array}$ & $\begin{array}{l}\text { F-value }=0.792 ; \mathrm{df}_{\text {denum }}=8 ; \mathrm{P}=0.532 \\
\text { F-value }=0.953 ; \mathrm{df}_{\text {denum }}=8 ; \mathrm{P}=0.46\end{array}$ \\
\hline $\begin{array}{l}\text { Total Leaf area } \\
\left(\mathrm{cm}^{2}\right)\end{array}$ & $\begin{array}{l}30 \\
60\end{array}$ & $\begin{array}{r}224.18 \pm 33.96 \mathrm{a} \\
333.41 \pm 103.73 \mathrm{a} \\
\end{array}$ & $\begin{array}{l}135.42 \pm 23.47 \mathrm{a} \\
222.25 \pm 16.24 \mathrm{a}\end{array}$ & $\begin{array}{l}117.59 \pm 20.59 b \\
276.30 \pm 36.75 a\end{array}$ & $\begin{array}{r}57.09 \pm 14.65 \mathrm{~b} \\
152.42 \pm 81.09 \mathrm{a}\end{array}$ & $\begin{array}{l}\mathrm{F} \text {-value }=8.148 ; \mathrm{df}_{\text {denum }}=8 ; \mathrm{P}=0.00815 \\
\text { F-value }=1.258 ; \mathrm{df}_{\text {denum }}=8 ; \mathrm{P}=0.352\end{array}$ \\
\hline
\end{tabular}

Note: means \pm SE followed by the same letter in the same rows were not significantly different according to LSD $\alpha=0.05$. $1=$ at the planting time; 2 = at the planting time and 1 -week after planting; $3=$ at the planting time, 1 -week and 2 -week after planting. The concentration of extract was $40 \%$

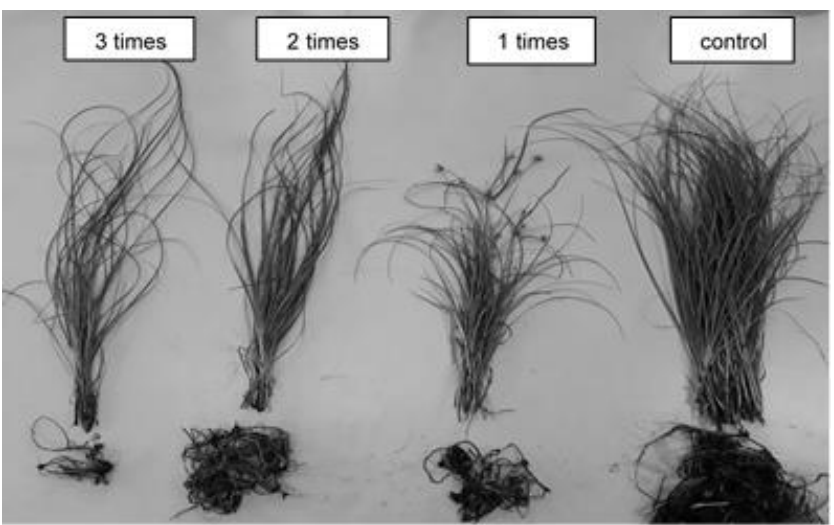

Figure 2. Growth of purple nutsedge foliage treated with different application times of cosmos flower extract at $60 \mathrm{DAS}$

\section{Chlorophyll content}

Chlorophyll a, b, and total in the purple nutsedge treated with the cosmos flower extract were only significantly lower than the control at 30 days after showing (Table 4), and there were no significant differences at 60 DAS.

\section{The photosynthetic rates}

Application of the cosmos flower extract reduced the photosynthetic rate (Table 5). Photosynthetic rates in the 2 application times of extract were significantly lower (Fvalue $\left.=0.678 ; \mathrm{df}_{\text {denum }}=8 ; \mathrm{P}=0.039\right)$ compared with the control at 30 DAS, but no significant differences were observed at 60 DAS $\left(\mathrm{F}\right.$-value $=0.148 ; \mathrm{df}_{\text {denum }}=8 ; \mathrm{P}=$ 0.928). 


\section{Dry weight}

The extracts significantly inhibited purple nutsedge foliage dry matter accumulation at 30 DAS. Foliage dry weight at 30 DAS in the treatment of 2 and 3 application times was significantly lower than those in the control, however, the two treatments were not significantly different (Table 6). All the dry matter was similar between the treated and the control at 30 DAS, but not at 60 DAS. The total dry weight was significantly decreased after extract applications. The highest inhibition was found at 3 application times after 30 days from sowing. However, there were no significant differences in purple nutsedge total dry matter accumulation after 60 DAS.

Table 3. N, P, K content (mg/dry weight) of purple nutsedge treated with different application times of cosmos flower extract.

\begin{tabular}{|c|c|c|c|c|c|c|}
\hline \multirow{2}{*}{$\begin{array}{l}\text { Nutrient } \\
\text { content }\end{array}$} & \multirow{2}{*}{ DAS } & \multicolumn{4}{|c|}{ Application Times } & \multirow{2}{*}{ F-value; $\mathbf{d f}_{\text {denum }} ; \mathbf{P}$} \\
\hline & & Control & 1 & 2 & 3 & \\
\hline \multirow{2}{*}{ Content of $\mathrm{N}$} & 30 & $15.41 \pm 0.76 \mathrm{a}$ & $12.40 \pm 1.76 \mathrm{ab}$ & $10.09 \pm 1.36 \mathrm{a}$ & $3.54 \pm 1.02 \mathrm{c}$ & F-value $=8.148 ; \mathrm{df}_{\text {denum }}=8 ; \mathrm{P}=7.74 \mathrm{e}-08$ \\
\hline & 60 & $11.08 \pm 1.80 \mathrm{a}$ & $8.32 \pm 0.42 \mathrm{ab}$ & $9.09 \pm 1.09 \mathrm{ab}$ & $5.03 \pm 1.51 \mathrm{~b}$ & F-value $=6.024 ; \mathrm{df}_{\mathrm{de}}$ \\
\hline \multirow{2}{*}{ Content of P } & 30 & $1.79 \pm 0.09 \mathrm{a}$ & $1.57 \pm 0.23 \mathrm{ab}$ & $1.21 \pm 0.16 b$ & $0.56 \pm 0.16 b$ & F-value $=6.906 ; \mathrm{df}_{\text {denum }}=8 ; \mathrm{P}=0.0131$ \\
\hline & 60 & $1.29 \pm 0.21 \mathrm{a}$ & $1.06 \pm 0.05 \mathrm{a}$ & $1.09 \pm 0.13 \mathrm{a}$ & $0.79 \pm 0.24 \mathrm{a}$ & F-value $=1.008 ; \mathrm{df}_{\text {denum }}=8 ; \mathrm{P}=0.438$ \\
\hline \multirow{2}{*}{ Content of K } & 30 & $16.98 \pm 0.84 a$ & $12.92 \pm 1.84 \mathrm{ab}$ & $10.73 \pm 1.45 b$ & $5.42 \pm 1.56 \mathrm{c}$ & F-value $=5.955 ; \mathrm{df}_{\text {denum }}=8 ; \mathrm{P}=0.0195$ \\
\hline & 60 & $12.20 \pm 1.98 \mathrm{a}$ & $9.66 \pm 1.15 \mathrm{a}$ & $8.68 \pm 0.44 \mathrm{a}$ & $7.68 \pm 2.31 \mathrm{a}$ & F-value $=0.947 ; \mathrm{df}_{\text {denum }}=8 ; \mathrm{P}=0.463$ \\
\hline
\end{tabular}

Note: means \pm SE followed by the same letter in the same rows were not significantly different according to LSD $\alpha=0.05$. $1=$ at the planting time; $2=$ at the planting time and 1-week after planting; $3=$ at the planting time, 1 -week and 2 -week after planting. The concentration of extract was $40 \%$.

Table 4. Chlorophyll a, b and total of purple nutsedge treated with different application times of cosmos flower extract

\begin{tabular}{|c|c|c|c|c|c|c|}
\hline \multirow{2}{*}{$\begin{array}{l}\text { Photosynthetic } \\
\text { pigments }\end{array}$} & \multirow[b]{2}{*}{ DAS } & \multicolumn{4}{|c|}{ Application Times } & \multirow{2}{*}{ F-value; $\mathbf{d f _ { \text { denum } } ; P}$} \\
\hline & & Control & 1 & 2 & 3 & \\
\hline \multirow{2}{*}{ Chlorophyll a } & 30 & $0.34 \pm 0.00 \mathrm{a}$ & $0.34 \pm 0.00 \mathrm{a}$ & $0.32 \pm 0.01 \mathrm{ab}$ & $0.26 \pm 0.040 \mathrm{~b}$ & F-value $=3.891 ; \mathrm{df}_{\text {denum }}=8 ; \mathrm{P}$ \\
\hline & 60 & $0.36 \pm 0.01 \mathrm{a}$ & $0.34 \pm 0.01 \mathrm{a}$ & $0.33 \pm 0.01 \mathrm{a}$ & $0.31 \pm 0.021 \mathrm{a}$ & F-value $=1.585 ; \mathrm{df}_{\text {den }}$ \\
\hline \multirow{2}{*}{ Chlorophyll b } & 30 & $0.23 \pm 0.00 \mathrm{a}$ & $0.24 \pm 0.01 \mathrm{a}$ & $0.21 \pm 0.01 \mathrm{ab}$ & $0.14 \pm 0.030 \mathrm{~b}$ & F-value $=5.897 ; \mathrm{df}_{\text {denum }}=8 ; \mathrm{P}=0.02$ \\
\hline & 60 & $0.27 \pm 0.02 \mathrm{a}$ & $0.22 \pm 0.02 \mathrm{a}$ & $0.24 \pm 0.01 \mathrm{a}$ & $0.20 \pm 0.030 \mathrm{a}$ & F-value $=1.383 ; \mathrm{df}_{\text {denum }}=8 ; \mathrm{P}=0.316$ \\
\hline Total & 30 & $0.26 \pm 0.00 \mathrm{a}$ & $0.26 \pm 0.00 \mathrm{a}$ & $0.24 \pm 0.01 \mathrm{ab}$ & $0.19 \pm 0.030 \mathrm{~b}$ & F-value $=4.566 ; \mathrm{df}_{\text {denum }}=8 ; \mathrm{P}=0.0381$ \\
\hline Chlorophyll & 60 & $0.28 \pm 0.01 \mathrm{a}$ & $0.25 \pm 0.01 \mathrm{a}$ & $0.26 \pm 0.02 \mathrm{a}$ & $0.24 \pm 0.020 \mathrm{a}$ & F-value $=1.308 ; \mathrm{df}_{\text {denum }}=8 ; \mathrm{P}=0.337$ \\
\hline
\end{tabular}

Note: means \pm SE followed by the same letter in the same rows were significantly different according to to LSD $\alpha=0.05$.

$1=$ at the planting time; $2=$ at the planting time and 1 -week after planting; $3=$ at the planting time, 1 -week and 2-week after planting.

The concentration of extract was $40 \%$.

Table 5. Photosynthetic rates $\left(\mu \mathrm{mol} \mathrm{m} \mathrm{m}^{-2} \mathrm{~s}^{-1}\right)$ of purple nutsedge treated with different application times of cosmos flower extract.

\begin{tabular}{|c|c|c|c|c|c|c|}
\hline & \multirow{2}{*}{ DAS } & \multicolumn{4}{|c|}{ Application Times } & \multirow{2}{*}{ F-value; $\mathbf{d f}_{\text {denum }} ; P$} \\
\hline & & Control & 1 & 2 & 3 & \\
\hline \multirow{2}{*}{$\begin{array}{l}\text { Photosynthetic } \\
\text { rates }\end{array}$} & 30 & $521.00 \pm 52.96 \mathrm{a}$ & $505.667 \pm 22.05 a$ & $481.331 \pm 4.06 \mathrm{ab}$ & $465.333 \pm 0.040 b$ & $\begin{array}{l}\text { F-value }=0.678 ; \mathrm{df}_{\text {denum }}=8 ; \\
P=0.039\end{array}$ \\
\hline & 60 & $641.67 \pm 108.85 a$ & $861.00 \pm 324.83 \mathrm{a}$ & $758.33 \pm 147.87 \mathrm{a}$ & $839.33 \pm 357.97 a$ & $\begin{array}{l}\text { F-value }=0,148 ; \mathrm{df}_{\text {denum }}=8 ; \\
\mathrm{P}=0.928\end{array}$ \\
\hline
\end{tabular}

Note: means \pm SE followed by the same letter in the same rows were not significantly different according to LSD $\alpha=0.05$. $1=$ at the planting time; $2=$ at the planting time and 1 -week after planting; $3=$ at the planting time, 1 -week and 2 -week after planting. The concentration of extract was $40 \%$.

Table 6. Dry weight (g/plant) of purple nutsedge foliage and underground organs treated with different application times of cosmos flower extract.

\begin{tabular}{|c|c|c|c|c|c|c|}
\hline \multirow{2}{*}{$\begin{array}{l}\text { Growth } \\
\text { characters }\end{array}$} & \multirow{2}{*}{ DAS } & \multicolumn{4}{|c|}{ Applications times } & \multirow{2}{*}{ F-value; $\mathbf{d f}_{\text {denum }} ; P$} \\
\hline & & Control & 1 & 2 & 3 & \\
\hline Dry weight of & 30 & $1.61 \pm 0.13 \mathrm{a}$ & $1.60 \pm 0.21 \mathrm{a}$ & $0.81 \pm 0.10 \mathrm{~b}$ & $0.42 \pm 0.10 \mathrm{~b}$ & F-value $=15.19 ; \mathrm{df}_{\text {denum }}=8 ; \mathrm{P}=0.00115$ \\
\hline foliage & 60 & $2.50 \pm 0.55 \mathrm{a}$ & $2.03 \pm 0.20 \mathrm{a}$ & $1.64 \pm 0.05 \mathrm{a}$ & $1.57 \pm 0.46 \mathrm{a}$ & F-value $=1.333 ; \mathrm{df}_{\text {denum }}=8 ; \mathrm{P}=0.33$ \\
\hline Dry weight of & 30 & $4.18 \pm 0.24 \mathrm{a}$ & $2.78 \pm 0.79 \mathrm{ab}$ & $2.75 \pm 0.49 \mathrm{ab}$ & $1.50 \pm 0.46 \mathrm{~b}$ & F-value $=4.25 ; \mathrm{df}_{\text {denum }}=8 ; \mathrm{P}=0.0452$ \\
\hline tuber & 60 & $1.68 \pm 014 \mathrm{a}$ & $1.31 \pm 0.08 \mathrm{a}$ & $1.18 \pm 0.10 \mathrm{a}$ & $1.15 \pm 020 \mathrm{a}$ & F-value $=0.406 ; \mathrm{df}_{\text {denum }}=8 ; \mathrm{P}=0.753$ \\
\hline Total dry & 30 & $5.79 \pm 0.29 \mathrm{a}$ & $4.38 \pm 0.62 \mathrm{ab}$ & $3.57 \pm 0.48 b$ & $1.92 \pm 0.55 \mathrm{c}$ & F-value $=10.44 ; \mathrm{df}_{\text {denum }}=8 ; \mathrm{P}=0.00386$ \\
\hline weight & 60 & $4.17 \pm 0.68 \mathrm{a}$ & $2.94 \pm 0.15 \mathrm{a}$ & $3.21 \pm 0.39 \mathrm{a}$ & $2.72 \pm 0.81 \mathrm{a}$ & F-value $=1.254 ; \mathrm{df}_{\text {denum }}=8 ; \mathrm{P}=0.353$ \\
\hline
\end{tabular}


In general, the application times did not significantly affect the purple nutsedge growth parameters, nutrient content, photosynthetic rates, and dry weight except for root numbers, the number of leaves/mother shoots/tuber, content of N, K, foliage dry weight, and total dry weight at 30 DAS. Root numbers, leaf numbers of mother shoots/tuber, and $\mathrm{N}$ content from samples treated with 3 application times of the extract were lower than 1 application time. The content of $\mathrm{K}$ decreased correspondingly as the application times increased. Purple nutsedge foliage dry weight from 2 and 3 application times, although not significantly different from each other, were significantly lower than 1 application time. Total dry weight from samples applied 3 times with cosmos flower extract was significantly lower from 1 and 2 application times.

\section{Discussion}

The results provide extensive information regarding the potential use of cosmos flower extracts as a bioherbicide against purple nutsedge beginning from the major compounds in the extracts, the effects on growth and physiological process of the purple nutsedge, chlorophyll content and nutrition absorption. The extract contained phenolic acid through phenolic's binding characteristic to ester contained in ethanol and form simple glycosides showed in the TLC test (Harborn 1996; Kozyra and Głowniak 2013). However, this study was able to demonstrate that cosmos contains gallic acid, a well-known allelochemical ( $\mathrm{Li}$ et al. 2010). Gallic acid has been reported to inhibit the germination of black gram (Vigna mungo) at $1 \mathrm{mM}$ (Suman et al. 2002). This suggests that the extract has the potential as a herbicide.

The cosmos flower extract variously inhibited purple nutsedge physical growth and physiological processes at 30 DAS, such as several growth parameters except for foliage growth, the reduction in $\mathrm{N}, \mathrm{P}$ and $\mathrm{K}$ content, chlorophyll content, photosynthetic rate, and dry weight accumulation. These results were consistent with previous research showing allelochemical effects (Abenovali et al. 2003; Bernat et al. 2007; El-Rokiek et al. 2010; Morais et al. 2014; Kaur and Sharma 2016). Phenol inhibited cytokinin activity, resulting in adverse cell division and plant growth (Isda et al. 2013). Nutrient uptake is influenced by the rate of transpiration, growth, nutrient content in the soil, and allelochemicals surrounding the roots. Nutrient absorption is affected by allelochemicals due to enzyme inhibition (Michelet and Bountry 1995; Morais et al. 2014). The enzyme $\mathrm{H}^{+}$-ATPase inhibits mineral extraction by roots, such as $\mathrm{N}$, and consequently causing detrimental effects on essential plant processes, such as photosynthesis, respiration or protein synthesis (Cheng and Cheng 2015). This enzyme is also responsible for the regeneration of proton electrochemical gradients (Michelet and Bountry 1995) promoting ion absorption and exchange, and metabolite drainage in plasma membranes (Palmgren 2001; Cheng and Cheng 2015). Nitrogen is the main constituent of chlorophyll and low levels of $\mathrm{N}$ due to biosynthesis disruptions of degradation enhancement affected chlorophyll levels causing lower photosynthetic rates (Taiz and Zeger 2002; Huang et al. 2010; Elisante et al. 2013; Poonpaiboonpipat et al. 2013). Leaf chlorophyll content is used as one of the parameters in understanding the response of plants to environmental stresses caused by allelochemical (Khang el al. 2016) resulting in lower dry weight accumulation (Elisante et al. 2013; Dadkhah 2015).

The purple nutsedge growth parameters, chlorophyll content, photosynthetic rates, and nutrition absorption of $\mathrm{P}$ and $\mathrm{K}$ were less sensitive to the extracts at 60 DAS. Older purple nutsedge were less sensitive against cosmos allelochemicals which may be caused by purple nutsedge developing resistances to allelochemicals. These results were consistent with previous findings where older weeds were less sensitive to allelochemical stress compared to younger ones (Gonzales et al. 1997). These suggest that purple nutsedge is less sensitivity against allelochemicals due to plant establishment.

It is noteworthy that Asteraceae contain other allelochemicals, such as cinnamic and benzoic acids, flavonoids, and various terpenes, which have been reported to be phytotoxic on crops and their usage should be monitored when used in agricultural fields (Singh et al. 2003; Li et al. 2010). This characteristic is important to reduce undesirable adverse effects of bioherbicides in agricultural system. The cosmos flower extract also inhibited the purple nutsedge growth due to its content, gallic acid. Further studies of gallic acid inhibitory mechanism are essential to fully understand the cosmos flower extract potential.

\section{ACKNOWLEDGEMENTS}

The research was supported by the Ministry of Research, Technology and Higher Education of the Republic of Indonesia.

\section{REFERENCES}

Abenavoli MR, Sorgona A, Sidari M, Badiani M, Fuggi A. 2003. Coumarin inhibits the growth of carrot (Daucus carota L. Cv. Saint Valery) cells in suspension culture. J Plant Physiol 160: 227-237.

Arora K, Batish DR, Singh HP, Kohli RK. 2015. Allelopathic potential of the essential oil of wild marigold (Tagetes minuta L.) against some invasive weeds. J Environ Agric Sci 3: 56-60.

Arunbabu T, Jena SN. 2018. Weeds and progressive weed management techniques in rice (Oryza sativa L.): a review. BEPLS 7: 108-117.

Batish, DR, Arora K, Singh HP, Kohli RK. 2007. Potential utilization of dried powder of Tagetes minuta as a natural herbicide for managing rice weeds. Crop Prot 26: 566-571.

Bernat W, Gawronska H, Zakresewska W, Gawronski SW. 2007. Physiological effects of allelopathic activity of sunflower on mustard. Allelopathy J 19: 1-10.

Bogatek R, Gniadzwoska A, Zakrzewska W, Oracz K, Gawronski SW. 2006. Allelopathic effects of sunflower extracts on mustard seed germination and seedling growth. Biol Plantarum 50: 156-158

Cheng F, Cheng Z. 2015. Research progress on the use of plant allelopathy in agriculture and the physiological and ecological mechanism of allelopathy. Front Plant Sci 6: 1-16.

Dadkhah A. 2015. Allelopathic potential of canola and wheat to control weeds in soybean (Glycine max). Russ Agric Sci 41: 111-114. 
Durigan JC, Timossi PC, Correia NM. 2006. Integrated management of purple nutsedge on sugarcane yield. Planta Daninha 24: 77-81.

Elisante F, Tarimo MT, Ndakidemi PA. 2013. Allelopathic effect of seed and leaf aqueous extracts of Datura stramonium on leaf chlorophyll content, shoot and root elongation of Cenchrus ciliaris and Neonotonia wightii. Am J Plant Sci 4: 2332-2339.

El-Rokiek KG, El-Din SAS, El-Masry RR. 2006. Improving the control of purple nutsedge (Cyperus rotundus L.) with glyphosate by preconditioning treatment with benzyl adenine. Egypt J Appl Sci 21: 79-96.

El-Rokiek KG, El-Din SAS, Messiha NK, El-Masry RR. 2009. Effect of growth regulators, alar or trichlorobenzoic acid and the herbicide basagran on the growth and propagative capacity of purple nutsedge (cyperus rotundus L). Ann Agric Sci 54: 59-75.

El-Rokiek KG, El-Masry RR, Messiha NK, Ahmed SA. 2010. The allelopathic effect of mango leaves on the growth and propagative capacity of purple nutsedge (Cyperus rotundus L.). Journal of American Science 6: 151-159.

El-Rokiek KG, El-Metwally IM, Messiha NK, El-Din SAS. 2007. Influence of some herbicides on the growth and propagative capacity of purple nutsedge (Cyperus rotundus L.). J Agric Sci 32: 2477-2489.

Ferell JA, Earl HJ, Vencill WK. 2004. Duration of yellow nutsedge (Cyperus esculentus) competitiveness after herbicide treatment. Weed Sci 52: 24-27.

Gonzales L, Sauto XC, Reigoza MJ. 1997. Weed control by Capsicum annum. Allelopathy J 4: 101-109.

Guha A, Sengupta D, Rasineni GK, Reddy AR. 2009. An integrated diagnostic approach to understand drought tolerance in mulberry (Morus indica L). Flora 205: 144-151.

Harborne JB. 1996. Metode Fitokimia: Penuntun Cara Modern Menganalisa Tumbuhan. Terbitan Kedua, ITB, Bandung. [Indonesian]

Hendrival ZW, Azis A. 2014. Periode kritis tanaman kedelai terhadap persaingan gulma. Jurnal Floratek 9: 6-13. [Indonesian]

Holm LG, Plucknett DL, Pancho JV, Herberger JP. 1991. The World's Sworst Weeds: Distribution and Biology. Krieger Pub Co. Malabar, FL, USA.

Horowitz M. 1992. Mechanisms of establishment and spreading of Cyperus rotundus - the worst weed of warm regions. Proc First Int Weed Cont Congr 1: 94-97.

Huang JH, Fu R, Liang CX, Dong DF, Luo XL. 2010. Allelopathic effects of cassava (Manihot esculenta Crantz.) on radish (Raphanus sativus L.) and ryegrass (Lolium perenne L.). Allelopathy J 25: 155-162.

Hussain I, Singh NB, Singh A, Singh H. 2017. Allelopathic potential of sesame plant leachate against Cyperus rotundus L. Ann Agrar Sci 15 141-147.

Hussain MI, Gonzalez L, Reigosa MJ. 2011. Allelopathic potential of Acacia melanoxylon on the germination and root growth of native species. Weed Biol Manag 11: 18-28.

Isda MN, Siti F, Rahmi F. 2013. Potensi ekstrak daun gulma babandotan (Ageratum conyzoides L.) terhadap perkecambahan dan pertumbuhan Paspalum conjugatum Berg. Jurnal Biologi 2: 120-125.

Kaisoon O, Siriamornpun S, Weerapreeyakul N, Meeso N. 2011. Phenolic compounds and antioxidant activities of edible flowers from Thailand. J Funct Food 3: 88-99.

Kamble PN, Sanjay PG, Ranjeet SM, Anupreet. 2015. Estimation of chlorophyll content in young and adult leaves of some selected plants. Univers J Environ Res Technol 6: 306-310.

Kaur G, Alamb MS, Jabbar Z, Javed K, Athar M. 2006. Evaluation of antioxidant activity of Cassia siamea flowers. J Ethnopharmacol 108: 340-348.

Kaur I, Sharma R. 2016. Allelopathic effect of Ageratum conyzoides on chlorophyll content in the leaves of mungbean. Int J Recent Sci Res 7: 13296-13297.
Khan R, Khan MA. 2010. Weed control efficiency of bioherbicides and their impact on grain yield of wheat (Triticum aestivum L.). EJAS. 4: 216-219.

Khang DT, Anh LH, Ha PTT, Tuyen PT, Quan NV, Minh LT, Quan NT, Minh TN, Xuan TD, Khanh TD, Trung KH. 2016. Allelopathic activity of dehulled rice and its allelochemicals on weed germination. ILNS 58: 1-10.

Kozyra M, Głowniak K. 2013. Phenolic acids in extracts obtained from the flowering herbs of Cirsium vulgare (Savi) Ten. growing in Poland. Acta Soc Bot Pol 82: 325-329.

Li ZH, Wang Q, Ruan X, Pan CD, Jiang DA. 2010. Phenolic and plant allelopathy. Molecules 15: 8933-8952.

Michelet B, Bountry M. 1995. The plasma membrane $\mathrm{H}^{+}$-ATPase: A highly regulated enzyme with multiply physiological functions. Plant Physiol 108: 1-6.

Moenandir J. 1993. Ilmu Gulma Dalam Sistem Pertanian. Raja Grafindo Persada, Jakarta.

Morais CSB, dos Santos LAS, Rossetto CAV. 2014. Oil radish development agronomic affected by sunflower plants reduces. Biosci J 30: 117-128.

Nishimoto R. 2001. Purple nutsedge tuber sprouting. Weed Biol Manag 1: 203-208.

Palmgren MG. 2001. Plant plasma membrane $\mathrm{H}^{+}$-ATPase: Powerhouse for nutrient uptake. Annu Rev Plant Biol 52: 817-845.

Poonpaiboonpipata T, Pangnakorna U, Suvunnameka U, Teerarakb M, Charoenyingc P, Laosinwattanab C. 2013. Phytotoxic effects of essential oil from Cymbopogon citratus and its physiological mechanisms on barnyard grass (Echinochloa crus-galli). Ind Crop Prod 41: 403- 407.

Shafique S, Bajwa R, Shafique S. 2011. Tagetes erectus L. - a potential resolution for management of Parthenium hysterophorus L. Pak J Bot 43: 885-894.

Shankar RM, Veeralakshmi S, Sirajunnisa AR, Rajendran R. 2014. Effect of allelochemicals from leaf leachates of Gmelina arborea on inhibition of some essential seed germination enzymes in green gram, red gram, black gram, and chickpea. Int Sch Res Notices 2014: 1-7.

Singh HP, Batish DR, Kaur S, Kohli RK. 2003. Phytotoxic interference of Ageratum conyzoides with wheat (Triticum aestivum). J Agron Crop Sci 189: 341-346.

Singh HP, Batish DR, Kohli RK. 2003. Allelopathic interaction and allelochemicals: new possibilities for sustainable weed management. Crit Rev Plant Sci 22: 239-311.

Sugiharti W, Trisyono YA, Martono E, Witjaksono. 2018. Manfaat bunga Turnera subulata dan Cosmos sulphureus bagi kehidupan Anagrus nilaparvatae (Hymenoptera: Mymaridae). JPTI 22: 43-50.

Suman A, Shahi HN, Singh P, Gaur A. 2002. Allelopathic influence of Vigna mungo (black gram) seeds on germination and radical growth of some crop plants. Plant Growth Regul 38: 69-74.

Taiz L, Zeger E. 2002. Plant Physiology. Third Edition. Sinauer Associates Inc. Publisher, Massachusets.

Webster TM, Grey TL. 2014. Halosulfuron reduced purple nutsedge (Cyperus rotundus) tuber production and viability. Weed Sci 62: 637646.

Wichittrakarn P. 2015. Allelopathic potential of Tagetes Erecta L.; its partially separation of active compounds and its mechanism on seed germination on Echinochloa Crus-galli (L.) Beauv. SICBENS-855

Yadav R, Bhullar MS, Kaur S, Kaur T, Jhala AJ. 2017. Weed control in conventional soybean with pendimethalin followed by imazethapyr+ imazamox/quizalofop-p-ethyl. Can J Plant Sci 97: 654-664.

Yang, CM, Chang, IF, Lin SJ, Chou CH. 2004. Effects of three allelopathic phenolics on chlorophyll accumulation of rice (Oryza sativa) seedlings: II. Stimulation of consumption-orientation. Bot Bull Acad Sin 45: 119-125.

Zeng Y, Zhao J, Peng Y. 2008. A comparative study on the free radical scavenging activities of some fresh flowers in southern China. LWTFood Sci Technol 41: 1586-1591. 